\title{
Proximity-Based Modelling of Cross-Contamination through Agent-Based Simulation: A Feasibility Study
}

\author{
Navonil Mustafee ${ }^{1}$, Korina Katsaliaki ${ }^{2}$, Eberhard E. Bischoff ${ }^{1}$, \\ Michael D. Williams ${ }^{1}$ \\ ${ }^{1}$ College of Business, Economics and Law, Swansea University, Swansea, UK \\ ${ }^{2}$ School of Economics and Business Administration, International Hellenic University, Thessaloniki, Greece.
}

\begin{abstract}
Proximity-based modelling methodology enables mathematical representation of a real system that is characterised by the existence of entities that come into physical contact. Healthcare systems can benefit from this methodology since physical proximity between entities (e.g., patients, clinical items like surgical equipment and blood units) can result in the spread of infectious diseases and cross-contamination. The existing analytical techniques, which are mainly based on differential equations, are unsuitable for representing the fine-grained, micro-world view of the entity interactions that we intend to model. We therefore extend Agent-Based Simulation (ABS) by enabling individual agents to be aware of the physical location of the other agents being modelled in a 3-Dimensional space - this is a perquisite for our proximitybased modelling methodology. To demonstrate the feasibility of our approach we experiment with a scenario wherein boxes of degradable clinical items, modelled as agents, are stored in close proximity. We use Cutting and Packing Optimisation (CPO) algorithms from literature to define the arrangement of these agents in the 3-D space and to make the individual agents "location-aware". An ABS model then simulates crosscontamination by modelling the spread of contaminants amongst the agents confined in the well-defined space. Our approach can be used to model analogous situations wherein physical proximity between entities in the underlying system is a necessary condition for entity interactions.
\end{abstract}

Keywords: Agents, Agent-Based Simulation, Container Loading Algorithm, Cutting and Packing Optimisation, Cross-Contamination, Proximity Modelling

\section{Introduction}

The healthcare problem scenario that forms the backdrop of this paper is the spread of infectious diseases and cross-contamination. Transmission of an infectious disease may occur by means of contact with infected individuals, by water, airborne inhalation, food, or through vector-borne spread. The control of the spread of infections such as HIV, MRSA, influenza, Mumps, West Nile virus, Tuberculosis (TB), Lassa virus and others, has been the aim of public policy and scientists for many decades. Similar, but additional, to the spread of infections among individuals, there is also the issue of cross-contamination of perishable items which are usually stored in close proximity. In the healthcare sector this problem is of high importance. There are several clinical items that have the possibility of cross-contamination during storage and/or transportation, such as, blood units (Vaught, 2006), donor organs (World Health Organisation, 2009), vaccines and medicines (World Health Organisation, 2003) and food provided to hospitalised patients (World Health Organisation, 2002). All of these items have limited shelf life and can potentially crosscontaminate other items. Using the right packaging method is one way to avoid cross-contamination. Clinical items should be stored individually and use the right packaging material and filling. They should also be kept at the right temperature. However, the storage and transportation of these items in close proximity is still an issue with regard to the problem of cross-contamination. This is even more prominent for food products (Ackerley et al, 2010) that are usually not as well packaged as clinical items due to the comparatively low purchase price of the former.

Mathematical models are an effective tool for investigating the dynamics of the spread of epidemics and cross-contamination, and subsequently to propose possible control strategies. Analytical techniques such as ordinary and partial differential equations have dominated this field for several decades; they model the system via causal connections at the macroscopic level (Emrich.et al, 2007). However, their practical use is often difficult, especially for predictive and quantitative analysis. The last decade has seen an alternative technique appeared on the stage, namely Agent-Based Simulation (ABS). This is a "bottom-up" approach focusing on the smallest unit of the system, an agent. Using the agent-based modelling and simulation approach researchers are able to do a preliminary "what-if" analysis with the purpose of assessing the system's behaviour under various conditions and evaluating which alternative policies to adopt (Bagni et al, 2002). 
In this research we propose the use of ABS with our proximity-based modelling methodology. Proximitybased modelling has been used previously to demonstrate that the combined use of GPS with RFID technology is technically feasible in determining the location of tagged materials (Song et al., 2007). However, this was not an ABS study; furthermore it considered only two dimensional spaces (thus, if the RFID-tagged materials were stacked on top of each other, it is arguable that this approach would not work). The objective of the present study is to extend ABS through use of intelligent location-aware agents, thereby facilitating the simulation of real systems that are characterised by the existence of entities that come in physical contact. We demonstrate the feasibility of our approach with regard to the aforementioned healthcare problem scenario - the cross-contamination of clinical items that are kept in close proximity during storage and/or transportation. For the purposes of this feasibility study we define "close proximity" as the requirement for physical contact between the clinical items being modelled. Thus, proximity-based modelling enables mathematical representation of a real system that is characterised by the existence of entities (e.g., patients, food items, blood units) that come in physical contact with each other.

This section has presented the motivation of this research. The remainder of this paper is structured as follows. Section 2 is devoted to ABS. The argument for using ABS for proximity-based modelling is presented in Section 3. Sections 4, 5 and 6 relate to the feasibility study (an overview of the study is presented in Section 4; Section 5 is devoted to the agent-based cross contamination model; Section 6 outlines the experiments and presents the results). Section 7 is the concluding section of this paper. It highlights the contribution of this work and draws the paper to a close.

\section{Agent-Based Simulation (ABS)}

ABS is a simulation technique that models the overall behaviour of a system under scrutiny through use of autonomous system components that communicate through transmission of messages. These autonomous and interacting system components are referred to as agents. The agents have behaviour and properties. The behaviour determines an agent's role in the environment and also its interactions with other agents. An agent's behaviour may change as the simulation progress through simulated time. Similarly, the agent properties also change during the course of the simulation, usually as a result of certain trigger points, for example, changes in the simulation time (in ABS the simulation time increments in discrete time steps.), changes triggered by an agent's internal state change, changes brought about as a result of messages being received from other agents, etc. Since there are usually a number of self-governing agents in an environment, each with the aforementioned characteristics, the overall system state is determined by the agents' dynamic interactions through time.

The description of agents and ABS that we presented above is based on our experience of working with this simulation technique. Charles. M. Macal and Michael. J. North have also discussed the characteristics of agents and $\mathrm{ABS}$ at length, and in the remainder of this paragraph we reference to a couple of their papers. Macal and North (2006) consider agents to have six specific characteristics, namely, attributes, behavioural rules, memory, resources, decision making sophistication and rules to modify behavioural rules. The agent behaviours, which are defined by simple rules, may be influenced by their interaction with other agents; and this "agent-by-agent and interaction-by-interaction" approach to modelling usually gives rise to emergent "patterns, structures and behaviours" that are not explicitly modelled in the system (Macal and North 2010).

The above discussions have made it apparent that ABS can be useful in modelling systems that comprise of several autonomous and interacting entities. However, in reality, these entities usually belong to certain broad categories of system constituents. For example, the system under investigation can be the electricity trading market, wherein there may be only two system constituents - electricity producers and electricity suppliers. However, there may be many producers of electricity (e.g., British Energy, Centrica Energy) and suppliers of electricity (e.g., British Gas, SWALES). In this example, the price of electricity is determined by the bargaining power of both the producers and suppliers. In modelling this scenario using ABS, the agents will usually be the individual producers and suppliers of energy (there may be hundreds of agents), however they will belong to either of the two system constituents (electricity producers and suppliers). Drawing parallels with the Object-Oriented Programming (OOP) terminology, each system constituent can be considered as a Class and each entity (agent) belonging to the system constituent can be considered as an Object of the Class. We purposely draw reference to OOP since we consider that it is easier to program ABS if we think of agents as objects. Indeed, a modeller trying to implement a complex ABS without objects may find, at the end of the modelling exercise, that she/he has unintentionally re-invented some of the OOPs concepts (Shaliz, 2006). 
ABS has several application areas. In financial trading, ABS has been used to model New Electricity Trading Arrangements (NETA) in the UK (Bunn and Oliveira 2001); application of this technique has been demonstrated in the financial market for price formation using realistic trade mechanism (Raberto et al. 2001). Agent-Based Social Simulation (ABSS) is the application of ABS in the social sciences context. Its use has been reported in the study of social dilemmas (e.g, Prisoner's Dilemma) by Gotts, Polhill, and Law (2003); Downing, Moss, and Pahl-Wostl (2001) report on the use of a prototype agent-based Integrated Assessment Model for understanding climate policy. ABS also has several applications in healthcare, and this is reviewed in the subsequent section that is devoted to literature review. ABS has been also applied in healthcare. Stainsby, Taboada, and Luque (2009) have used agents to model hospital emergency departments; Sibbel and Urban (2001) have integrated agent-based approaches into classical simulation systems to enable better hospital management. The reader is referred to Mustafee et al. (2010) and Katsaliaki and Mustafee (2011) for further discussion on ABS in the context of healthcare (including its application compared to other simulation techniques like discrete-event simulation, Monte Carlo simulation and system dynamics).

\section{Proximity-Based Modelling in Healthcare: Why ABS?}

Much research has been done in recent years in the development of mathematical and computer models of epidemics (Halloran et al., 2002; Eubank et al., 2004, Ferguson et al., 2006; Coburn et al., 2009). These have greatly contributed to the understanding of the spread of infectious disease and the measures needed to control or mitigate them. Most of these studies use computer simulation models to mimic the behaviour of the spread and have and will play an important role in evaluating containment and mitigation strategies for future epidemics (Halloran et al., 2008). These models usually include age structure, social groups and social contact networks among individuals that dynamically evolve over time and adapt to actions taken by individuals and public health interventions. These tools differ in their underlying modelling approaches and in the implementation, flexibility, and accessibility of the software itself. Most of these approaches (with the exception of ABS - this is discussed in the next paragraph) are unsuitable for supporting the proximity-based interactions taking place between entities in an underlying physical system; these interactions are triggered only when the entities come in physical contact in the 3-D space.

Agents are used in modelling the spread of infectious diseases with the capability of tracking the movement of a disease and the contacts between each individual in a social group located in a geographic area (Bagni et al., 2002; Patlolla et al., 2004). Agent-based models describe the stochastic propagation of a disease at the individual level, thus taking into account the explicit social and spatial structure of the population under consideration (Gordon, 2003; Dunham, 2005; Hotchkiss et al. 2005). By modelling the correlations between individuals, it is possible to understand the role of spatial heterogeneity in spreading dynamics. Different ABS models have been developed in order to depict the spatial behaviour of diverse infectious diseases through structured and realistic urban networks (Patlolla et al., 2004; Eidelson et al., 2004; Carley et al., 2006).

The discussion on ABS in sections 2 and 3 has shown that the use of agents enable us to model individual entities. This allows agent-based analysis of social and organisational systems (which may be composed of either humans or are artificial in nature) with the objective of further understanding the principles of coordination and management among agents engaged in real tasks (CASOS, 2012). A conventional ABS model can generally be created for such analysis. In a conventional ABS model the agent interactions are usually defined by the modeller using package-specific functionalities. For example, AnyLogic, defines four predefined agent layouts in continuous space (random, ring, arranged and spring mass layouts) and two layouts in discrete space (random and arranged layouts) - these layouts are depicted in Figure 1 below (XJ Technologies, 2011). The agents are represented as dots or squares of different colours (red, black, blue) and the agent relationships are indicated by either lines (in case of agents in continuous space) or the physical placement of the agents (in case of agents in discrete space).

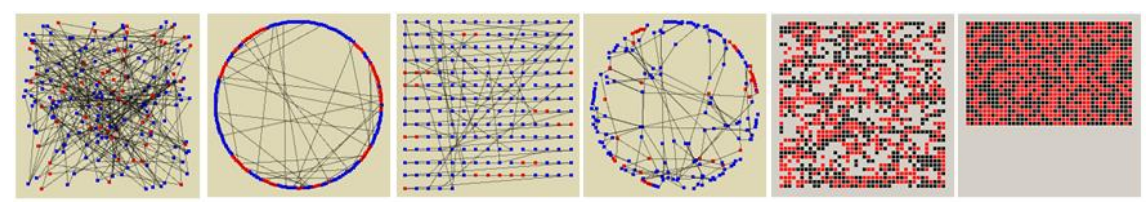

Figure 1: AnyLogic-defined arrangement of agents (from left to right): Random layout, Ring layout, Arranged layout, Spring Mass layout, Random layout (discrete space), Arranged layout (discrete space) (XJ Technologies, 2011).

This pre-defined agent arrangement and the pre-defined inter-agent relationship is unsuitable for our proximity-based modelling approach. Thus, we extend ABS by proposing the creation of intelligent location- 
aware agents in 3-D space. Figure 2 visually depicts our approach. Every agent has a pre-defined physical dimension (length, breadth and height). Every agent is aware of its location (for example, the agent depicted by the red box in Figure 2 is aware that it is surrounded by another four boxes on its side - thus inter-agent relationship is created based on physical proximity with other agents). Every agent has a colour attribute (this is only to increase the visual appearance and for error-checking; it has no significance with regard to the proposed methodology). We term these agents as being "location-aware"; these "location-aware" agents are essential for the implementation of our proximity-based modelling methodology through use of ABS.

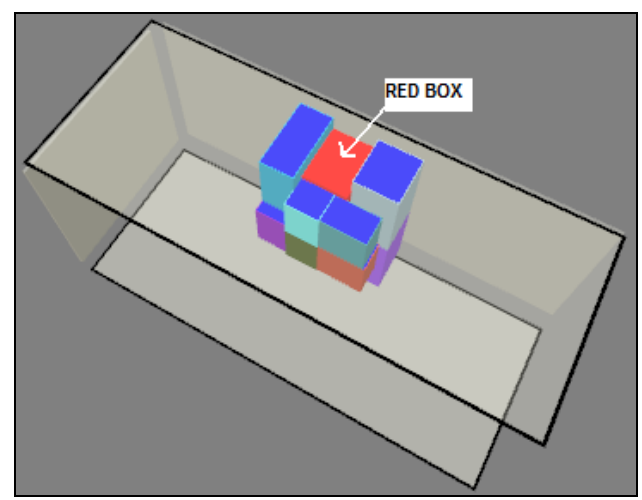

Figure 2: Establishing inter-agent relationship based on physical proximity between agents

Sections 4, 5 and 6 are devoted to the feasibility study, the objective of which is to demonstrate the application of our stated methodology for modelling of cross-contamination.

\section{Modelling of Cross-Contamination through use of Location-Aware Agents: A Feasibility Study}

The feasibility study describes the scenario wherein boxes of degradable items are stored in close proximity. In the healthcare context, these boxes may contain blood units, donor organs, vaccines and medicines or even patients' food supplies - all of which have limited shelf life and can potentially cross-contaminate other items. Thus, as some of these items deteriorate over time these results in the boxes becoming contaminated and, in turn, cross-contaminating other boxes that are in direct contact with it.

As is evident in literature (Bhat, 1988), the value of perishable food products will decay once they are produced, and different kinds of perishable products may have different decay rates. Studies from food microbiology state the high probability of agricultural commodities to be damaged by mould growth and subsequent aflatoxin accumulation during transit (Harding and Dyre, 1942; Bhat, 1988). For the problem of perishable goods, a supplier usually cares more about the decay of perishable goods than the average loading ratio and is willing to use more vehicles to prevent the deterioration (Chen et al., 2009). Moreover, It is clear that road conditions causes the physical damage to the fresh produce and non-removal of foreign bodies causes perishability (Acharyulu and Madhavedi, 2011). In addition, contamination and cross-contamination may occur in the shipment of not disinfected medical devices. This risk occurs when liquids from the used medical device seep out of the device and into the packaging case and then out of it (Robinson and McLeod, 1989).

The feasibility study will investigate the trade-off between optimisation of physical space for storage/transportation (e.g., stockroom or warehouse stocking clinical items) and the potential for crosscontamination among boxes containing perishable clinical items.

In this study we have used a variant of the Cutting and Packing Optimisation (CPO) algorithms, namely, Container Loading Algorithms (CLAs), in order to automate the placement of the boxes (location-aware agents) in the 3-D space and to assign them the attributes of length, breadth and height. A comprehensive literature review is provided by Pisinger (2002), and Wäscher, Haußner, and Schumann (2007) which discuss CLAs in relation to the wider literature on CPO. Using CLAs allows us to experiment with multiple layouts pertaining to the location-aware agents (Figure 3) and this, in turn, informs us of the trade-off between optimisation of physical space and the potential for cross-contamination. The experiments are described in Section 6. 


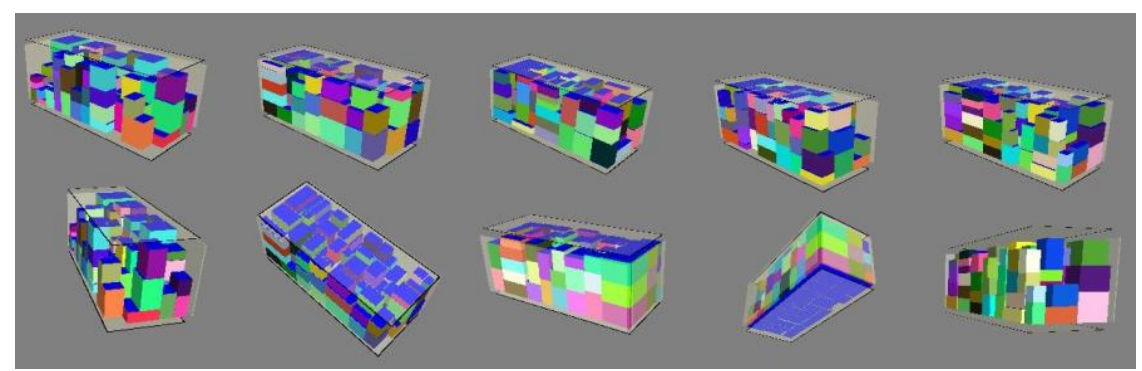

Figure 3: $\mathrm{CPO}$ algorithm being used to generate various layouts for the location-aware agents and to create inter-agent relationships. The agents are represented by the individual boxes in the confined space (AnyLogic visualisation).

\section{Implementation of the ABS Model}

The CLA-generated layouts (refer to Section 4) are imported into the AnyLogic agent-based modelling environment. As mentioned earlier, the most important element of our methodology is the creation of interagent relationships based on proximity-based modelling; this is entirely dependent on the output generated by the CLAs. The CLA output (Figure 4) provides the X, Y and Z coordinates for every box, and these coordinates are used to not only generate a 3-D representation of the box in a confined space but also to establish agent relationship amongst the boxes (through use of Java programming language and AnyLogic libraries - refer to Table 1 for the code related to the creation of inter-agent relationships). We refer to this as proximity-based agent modelling since these relationships are established only when the physical personifications of the agents (each agent is represented as a box and it has well-defined length, breadth and height attributes) come in contact with each other. As can be seen in Figure 2 above, a randomly-selected agent (red box) is related to all the eight boxes that it is in physical contact with. Thus, the agent represented by the red box will be able to send messages to all the surrounding boxes, and each of these boxes will likewise be able to communicate with its neighbours, and so forth.

\begin{tabular}{|c|c|c|c|c|c|c|c|c|}
\hline 1 & 587 & 233 & 220 & \multicolumn{4}{|c|}{ Physical space dimensions } & \multirow{3}{*}{$\begin{array}{l}\text { Number of } \\
\text { items }\end{array}$} \\
\hline 2 & 66 & \multicolumn{6}{|l|}{ t } & \\
\hline 3 & 0 & 0 & 0 & 99 & 120 & 73 & 57 & \\
\hline 4 & 0 & 120 & 0 & 120 & 193 & 99 & 57 & \multirow{15}{*}{$\begin{array}{l}\text { Freshness } \\
\text { Index }\end{array}$} \\
\hline 5 & 99 & 0 & 0 & 198 & 120 & 73 & 57 & \\
\hline 6 & 120 & 120 & 0 & 240 & 193 & 99 & 57 & \\
\hline 7 & 198 & 0 & 0 & 271 & 120 & 99 & 57 & \\
\hline 8 & 240 & 120 & 0 & 339 & 193 & 120 & 57 & \\
\hline 9 & 271 & 0 & 0 & 375 & 74 & 65 & 24 & \\
\hline 10 & 339 & 74 & 0 & 413 & 178 & 65 & 24 & \\
\hline 11 & 375 & 0 & 0 & 440 & 74 & 104 & 24 & \\
\hline 12 & 413 & 74 & 0 & 478 & 178 & 74 & 24 & \\
\hline 13 & 339 & 178 & 0 & 420 & 233 & 92 & 18 & \\
\hline 14 & 420 & 178 & 0 & 501 & 233 & 92 & 18 & \\
\hline 15 & 478 & 0 & 0 & 559 & 92 & 55 & 18 & \\
\hline 16 & 478 & 92 & 0 & 559 & 147 & 92 & 18 & \\
\hline \multirow[t]{2}{*}{17} & 501 & 147 & 0 & 556 & 228 & 92 & 18 & \\
\hline & \multicolumn{3}{|c|}{$X, Y$ and $Z$ co-ordinates } & \multicolumn{4}{|c|}{ Length, Breadth and Height } & \\
\hline
\end{tabular}

Figure 4: Output of the Container Loading Algorithm (Bischoff, 2006); used as input for the creation of inter-agent relationship in our ABS model

Table 1: Code for establishing inter-agent relationship between agents (please note the code has been simplified)

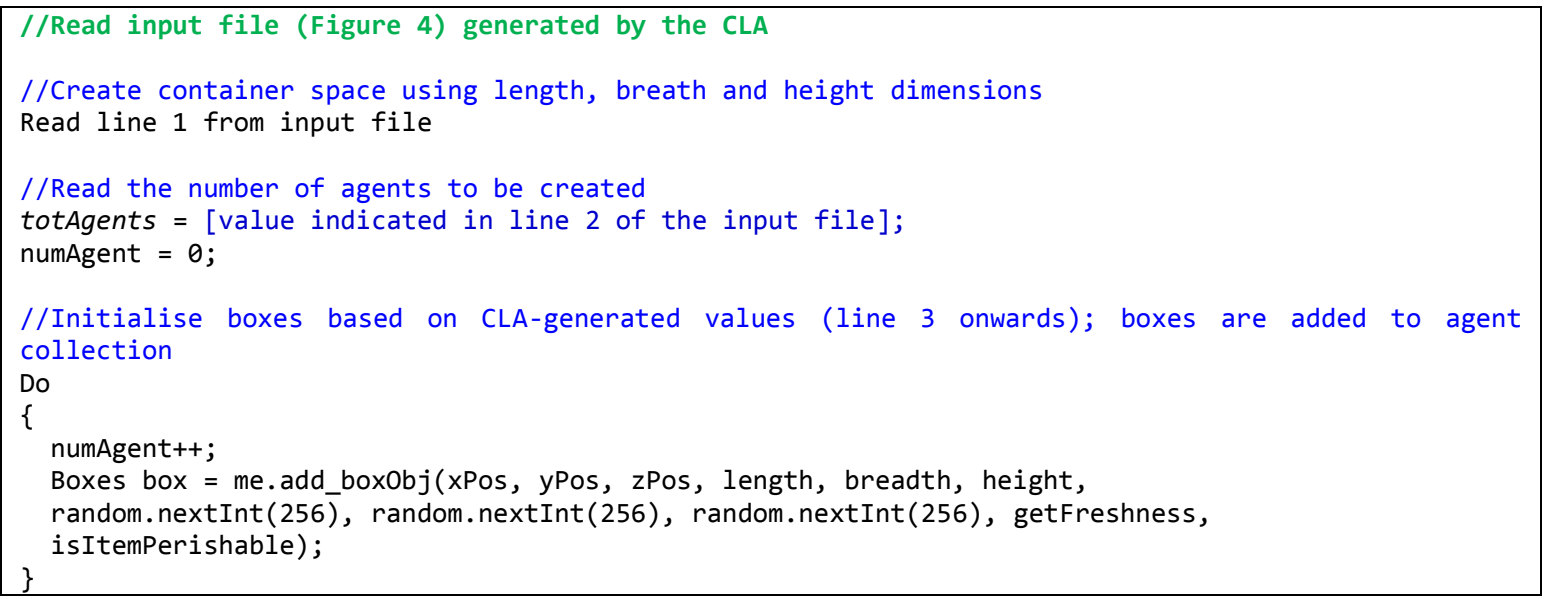




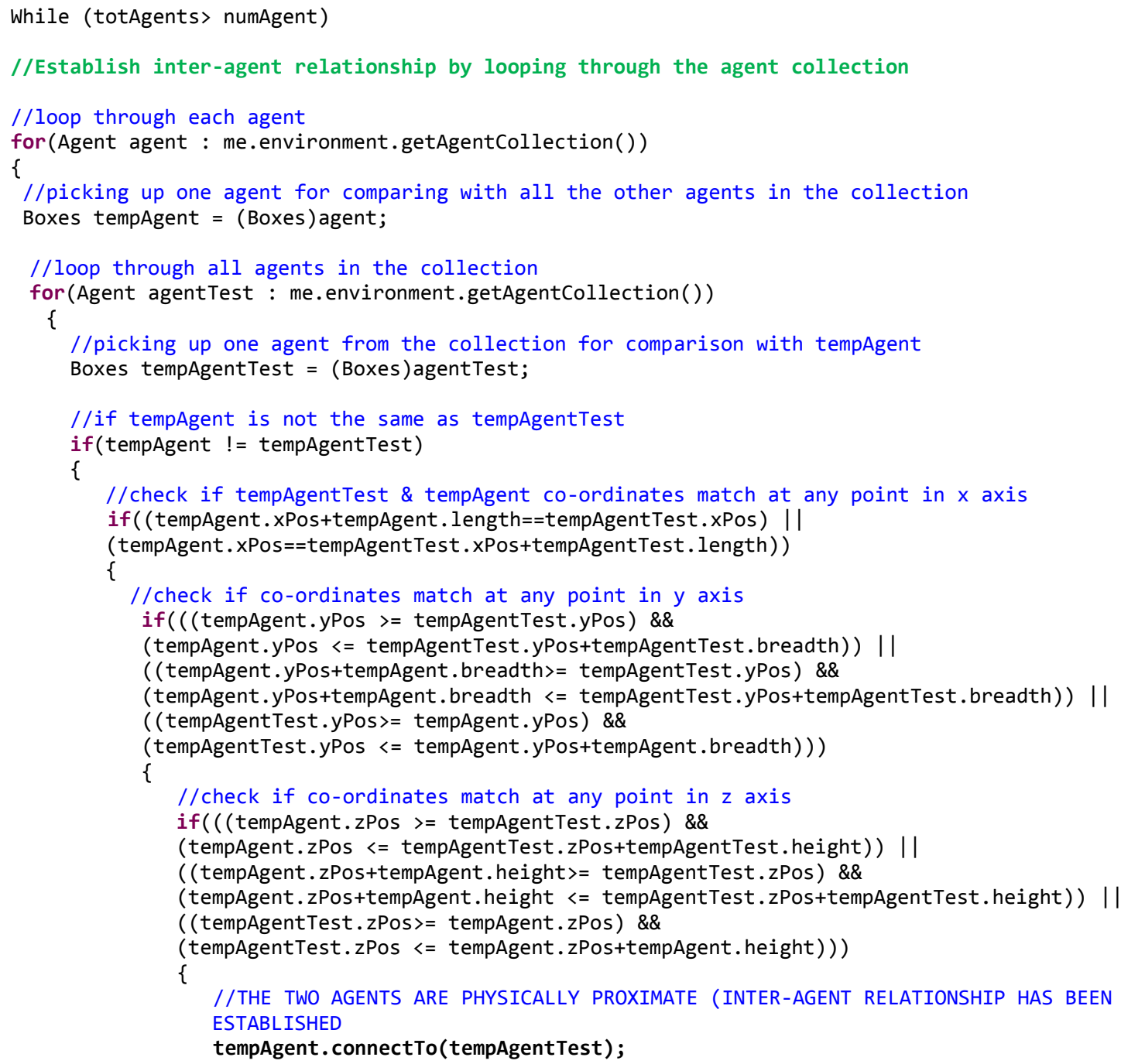




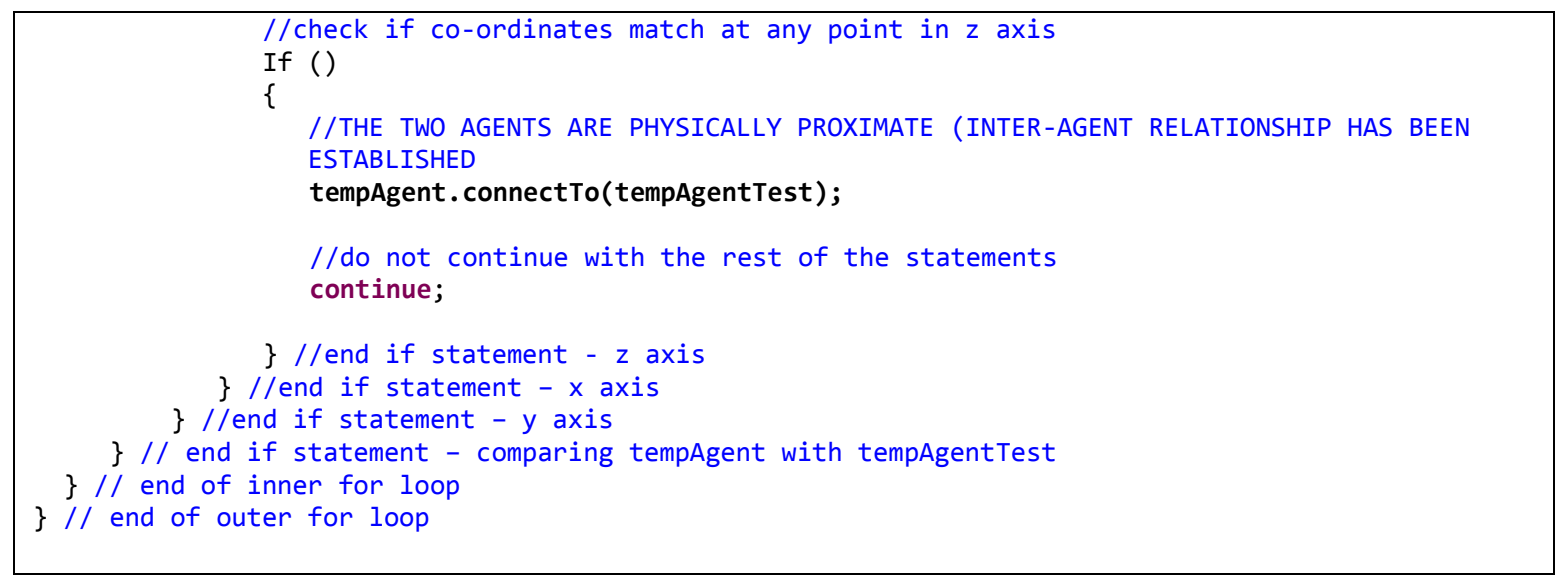

The modelling and propagation of contaminants is now discussed. Every agent has the property freshness index that is included in the CLA output (the other agent properties are length, breadth, height, xPos, yPos, zPos, lineRed, lineGreen, lineBlue; refer to Table 2 for further explanation of these properties). This index is a randomly CLA-generated value between 5 and 60 and it is assigned to different box types, i.e. all boxes of the same type have the same freshness index. The index is used to denote the number of simulated days for which the contents of the box will remain contamination free. This is not necessarily related to the units' expiration date ("best before" or "use by" date). The boxes become contaminated when moulds start to develop. Thus, as the simulation progresses in time, at the end of every simulated day, the freshness value associated with every agent is decreased by one (if freshness $>0$ ). In the model we have assumed that when the freshness level of a particular box reaches zero, it is mould affected. The transition of the agents is modelled by a state chart (Figure 5 - left). At the start of the simulation the state of every agent is "StateFresh". Following the logic of the simulation and the modelling assumption stated earlier, when the freshness value of an agent becomes zero, the agent state changes from "StateFresh" to "StateMould" and it sends messages to all the agents with which it is in physical contact (Figure 5 - sendMessagetoAgents) to indicate its change of state.

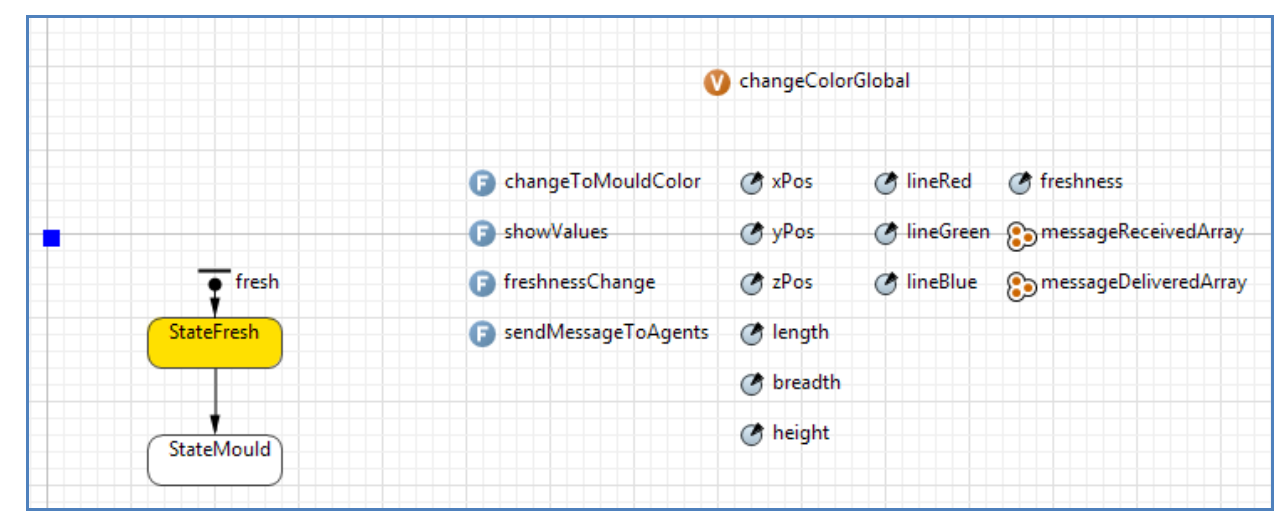

Figure 5: Agent state chart (left) and the variables and functions used to realise the model

We have used freshness index in our simulation since there is evidence in literature of contamination and/or decay with regard to perishable products subsequent to the expiration of well-defined time units; also, the time units may differ from one product to the other. For example, the mould infection (fungal spoilage) for fruits in transit for up to 14 days can be as high as $100 \%$ with the average mould rate depending on the fruit being transferred (Tournas and Katsoudas, 2005). Decay fungi are easily spread throughout the shipping container (Ashby, 1995) and at different rates, for example, berries in a shipping container have an average mould rate of $80 \%$ in 14 days of transit, citrus fruits have an average of $45 \%$, etc. (Tournas and Katsoudas, 2005). In our case we indicate that contamination will occur in between 5 to 60 days of shipment; when this happens the colour of the box is changed in the 3-D space so as to enable easier identification (Figure 6). Our cross-contamination model is executed for 30 simulated days. 


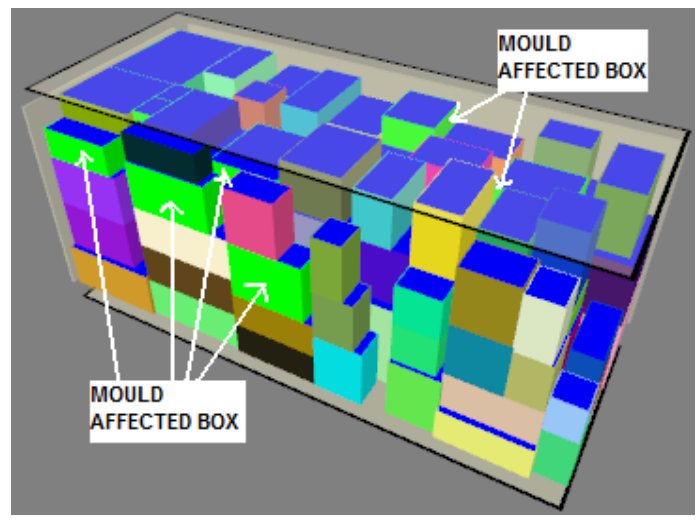

Figure 6: Boxes get cross-contaminated through the propagation of mould as the simulation advances through time

Soon after a box has become affected by mould, it starts to contaminate other boxes which physically surround it. This contamination is spread in the model by the proximity-based interactions sent by the mouldaffected agent to all the surrounding agents. Thus, in Figure 2 above, if the red box/agent were to become mould-affected, it would immediately send messages to all the eight boxes/agents surrounding it. The message is a signal to the other agents (each of whom has its own copy of freshness index that decreases over time) that they have now been contaminated. Upon receiving this message, each agent immediately decreases its existing value of freshness index by 1 (if freshness index $>0$ ). This is in addition to the drop of 1 unit of freshness that is applied at the end of each simulated day. Since the agents represent boxes that are in physical contact with each other (and therefore susceptible to cross-contamination), it is therefore the case that, following the logic of the program the majority of the boxes will be contaminated before the expiry of their freshness index. This is best demonstrated in a subsequent figure (Figure 7), which shows that irrespective of the box layout pattern used, $60-80 \%$ of the boxes will become mould affected by the end of the 30-day simulated run.

The logic of the model, as described above, is repeated until the end of the simulation period. Several pieces of data are then collected, including the number of mould-affected boxes, the maximum freshness value associated with an item of cargo, average cargo freshness, etc. This completes a single ABS simulation of one CLA-generated layout pattern. Several such simulations have to be executed, each with a different CLAgenerated boxes' layout pattern, in order to investigate the trade-off between optimisation of physical space for storage/transportation and the potential for cross-contamination.

Although it may be argued that the individual items in our simulation could have been modelled using Object Oriented Discrete-Event Simulation, this was not considered for several technical and methodological reasons (e.g., our scenario could not be "intelligently" modelled as a network of queues and servers; our requirement for asynchronous execution of individual object instances). Considering also the fact that $\mathrm{ABS}$ reinforces object oriented conceptualisation and modelling (Shaliz, 2006) of the problem in terms of Classes and instances (objects) of the Classes, our chosen simulation technique is arguably appropriate for the scenario presented. The individual items in our simulation are best modelled as agents because they display three important characteristic consistent with most agents - (1) they have properties, (2) they exhibit autonomous behaviour, and (3) they interact with other agents and this leads to more complex behaviour. Table 2 lists the three agent characteristics (column one) in relation to the cross-contamination scenario discussed in the paper (column two).

Table 2: Agent characteristics for the cross-contamination scenario

\begin{tabular}{|c|c|}
\hline Agent Characteristics & Cross-Contamination Scenario \\
\hline $\begin{array}{l}\text { (1) Agents have } \\
\text { properties }\end{array}$ & $\begin{array}{l}\text { - Property: Freshness index } \\
\text { - Dimension of the individual items of cargo (properties: length, } \\
\text { breadth, height) and their X, Y, Z coordinates in the 3-D space } \\
\text { (properties: xPos, yPos, zPos) - these six variables are used to } \\
\text { determine which boxes that are in physical contact and the } \\
\text { resultant inter-agent relationships. } \\
\text { - RGB color scheme of the boxes (properties: lineRed, lineGreen, } \\
\text { lineBlue). }\end{array}$ \\
\hline $\begin{array}{l}\text { (2) Agents exhibit } \\
\text { autonomous behavior }\end{array}$ & $\begin{array}{l}\text { - The freshness index will decreases over time. } \\
\text { - When the freshness attribute reaches zero, the state of an agent } \\
\text { will change from "StateFresh" to "StateMould". }\end{array}$ \\
\hline
\end{tabular}




\begin{tabular}{|l|l|}
\hline $\begin{array}{l}\text { (3) Interaction between } \\
\text { agents may result in } \\
\text { more complex behavior }\end{array}$ & $\begin{array}{l}\text { Perishable items will be cross-contaminated when they come in } \\
\text { physical contact with other mould-effected items having } \\
\text { freshness index }=0 \text { (or, state= "StateMould"); }\end{array}$ \\
$\begin{array}{l}\text { In other words, the "StateMould" agents will interact with the } \\
\text { other "StateFresh" agents in physical proximity; this inter-agent } \\
\text { interaction determines the rate at which mould spreads through } \\
\text { the confined space. }\end{array}$ \\
\hline
\end{tabular}

\section{Experiments and Results}

For our cross-contamination experiments we have employed the CLA originally proposed by Bischoff (2006). Both the items and the three dimensional spaces are assumed to be rectangular in shape and have known dimensions (in Figures 2, 3 and 6 the space constraint is depicted using transparent walls that surround the boxes). The items ("boxes") are assumed to belong to different "box types". The predominant factor that distinguishes the various box types are the boxes' physical dimensions. In our experiments we have used 20 different box types (thus, we may have a total of 200 individual boxes; however, these boxes will belong to one of the 20 box types). Finally, for the purpose of the cargo cross-contamination scenario presented in this paper we have introduced a new property - the freshness index (see section 5).

Several experiments are required to be conducted in order to find the trade-off between the optimisation of physical space and the potential for cross-contamination. In our feasibility study the number was limited to 100. In other words, using the CLA algorithm (Bischoff, 2006) we generated 100 different box layout patterns for our experiments. Each box layout pattern had a corresponding space utilisation percentage. We ranked these utilisation rates and selected the following five:

(a) Layout pattern with the lowest space utilisation (BTMmin);

(b) Layout pattern with the highest space utilisation (BTMmax);

(c) Layout pattern with space utilisation percentile rank of 25 (BTM25);

(d) Layout pattern with space utilisation percentile rank of 50 (BTM 50$)$;

(e) Layout pattern with space utilisation percentile rank of 75 (BTM75).

Figure 7 shows the percentage of mould-affected items for the five different space utilisation patterns (BTMmin; BTMmax; BTM25; BTM 0 ; BTM75). The graph shows the existence of trade-offs between space utilisation and the percentage of boxes affected with mould. The five layout patterns selected for simulation had utilisation efficiencies of 58.79\% (BTMmin), 78.93\% (BTM 25$), 82.60 \%$ (BTM 50$), 84.43 \%$ (BTM 75 ) and 89.94\% (BTM $\max$ ), respectively; the corresponding proportions of mould-affected boxes were $65.15 \%$, $72.22 \%, 70.65 \%, 69.79 \%$ and $76 \%$. Thus, changes in space utilisation rates (brought about by a change in the box layout plan) do have an effect on the percentage of items that are spoilt.

In an ABS the behaviour of the system emerges from the interaction of the individual agents. Although, intuitively, it would seem that a layout pattern with the greater space utilisation (e.g., BTM75) would result in more number of mould-affected boxes (when, for example, compared to BTM25), however, there are several factors which determine the $\%$ of boxes affected. One such factor is the number of boxes used in the layout pattern. To take an extreme example, we can have a case where only 5 large boxes have been identified by the CLA to achieve 95\% space utilization; in contrast, in another layout, the CLA identifies 500 small boxes and achieves $87 \%$ space utilization. Since every box has a freshness index and when it reaches zero it can cross-contaminate the surround boxes, it therefore follows that the spread of mould in the latter layout (with $87 \%$ space utilization) will arguably be more amplified due to the fact that in this arrangement there are many boxes that are in contact with each other. Another reason for this can be the freshness index associated with boxes that are selected by the CLA algorithm. As has been mentioned in the paper, the freshness index is a randomly generated value between 5 and 60 and it is assigned to different box types, i.e. all boxes of the same type have the same freshness index. Thus, the propagation of mould also directly depending on the freshness attribute of specific boxes being selected by the CLA algorithm - and this may change from one layout plan to the other.

The results show the absence of either positive or negative correlation between container utilisation percentage and the $\%$ of boxes affected with mould. For example, the $\%$ of mould affected boxes would roughly be the same for $\mathrm{BTM}_{25}, \mathrm{BTM}_{50}$ and $\mathrm{BTM}_{75}$ layout patterns, although there is an increase in their respective space utilisation percentages. 


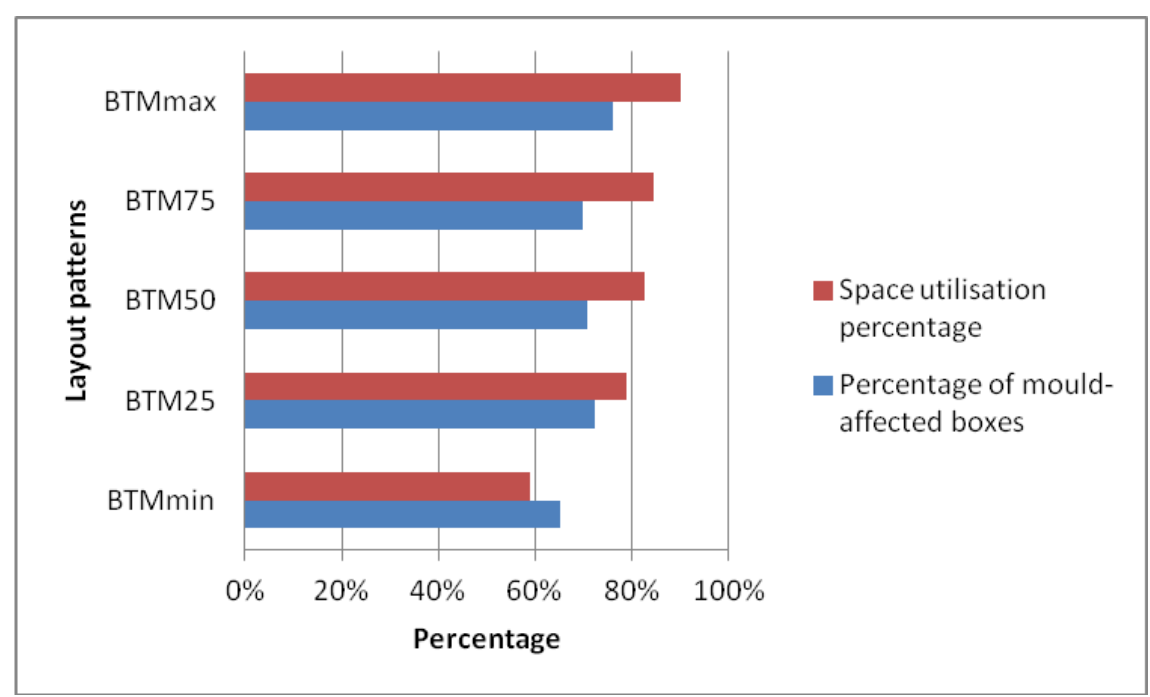

Figure 7: Percentage of mould-affected boxes for different space utilisation percentages

The results indicate the opportunity for a trade-off. For example, the stakeholder, having seen the results of five experiments, may decide to use the layout pattern that provides $84.43 \%$ utilisation efficiency (BTM75) and not BTMmin which provides 58.79\% efficiency but has the lowest percentage of boxes affected with mould. This may be because with the BTM 75 box layout plan the stakeholder will be able to transport more boxes (96 as against 66 if he chose BTM75), and this may make up for the slight increase in the possible degree of contamination for the chosen scheme.

Table 3 presents freshness values (divided into well-defined bin ranges) for the experiments that were performed. It shows that for all the five loading plans that were simulated, between 65 to $76 \%$ of items had already developed mould or would be mould affected in the next three days (note the freshness/bin range: 0.00-3.00). It further shows that if BTMmin loading plan is selected, then approximately $33.33 \%$ of items will remain relatively fresh (bin/freshness range: 15.00 to 30.00) by the end of the simulation - and this value is at least $50 \%$ more when compared to the corresponding freshness values of the alternate loading plans. Here again there may be an opportunity for a trade-off!

Table 3: Data showing freshness percentages corresponding to the simulation experiments

\begin{tabular}{|c|c|c|c|c|c|}
\hline BIN RANGE & BTMmin & BTM25 & BTM50 & BTM75 & BTMmax \\
\hline $0.00-3.00$ & $65.15 \%$ & $72.22 \%$ & $70.65 \%$ & $70.83 \%$ & $76.00 \%$ \\
\hline $3.01-6.00$ & $0.00 \%$ & $3.33 \%$ & $0.00 \%$ & $2.08 \%$ & $0.00 \%$ \\
\hline $6.01-9.00$ & $0.00 \%$ & $1.11 \%$ & $4.35 \%$ & $3.13 \%$ & $1.00 \%$ \\
\hline $9.01-12.00$ & $0.00 \%$ & $0.00 \%$ & $0.00 \%$ & $0.00 \%$ & $0.00 \%$ \\
\hline $12.01-15.00$ & $1.52 \%$ & $2.22 \%$ & $2.17 \%$ & $4.17 \%$ & $6.00 \%$ \\
\hline $15.01-18.00$ & $7.58 \%$ & $5.56 \%$ & $6.52 \%$ & $7.29 \%$ & $5.00 \%$ \\
\hline $18.01-21.00$ & $10.61 \%$ & $6.67 \%$ & $10.87 \%$ & $8.33 \%$ & $8.00 \%$ \\
\hline $21.01-24.00$ & $10.61 \%$ & $5.56 \%$ & $3.26 \%$ & $3.13 \%$ & $2.00 \%$ \\
\hline $24.01-27.00$ & $1.52 \%$ & $1.11 \%$ & $2.17 \%$ & $1.04 \%$ & $2.00 \%$ \\
\hline $27.01-30.00$ & $3.03 \%$ & $2.22 \%$ & $0.00 \%$ & $0.00 \%$ & $0.00 \%$ \\
\hline Total Boxes & $100.00 \%$ & $100.00 \%$ & $100.00 \%$ & $100.00 \%$ & $100.00 \%$ \\
\hline
\end{tabular}

\section{Conclusion}

In the healthcare sector there are numerous examples of clinical items that degrade over time and can potentially contaminate other items, for example, clinical waste, blood, donor organs, and medicines. The work presented in this paper has combined our proximity-based modelling approach with ABS for modelling cross-contamination amongst such items. The feasibility study focussed on items that are stored or transported in close proximity with the objective of finding trade-off between the optimisation of physical space and the potential for cross-contamination.

Although it can be argued that wastage due to cross-contamination can be substantially reduced by taking adequate precautions during storage and transportation (e.g., making sure that clinical items are kept 
segregated, providing refrigerated storage spaces), it is important to recognise the underlying cost implications. Let us take the example of policies like segregation of items during storage. The average cost associated with storing and transporting items will usually increases as a result of such policies since the utilisation of storage / container space will be reduced. On the other hand, although the optimal utilisation of storeroom and container space will reduce the per-unit cost associated which storage and transportation, this may lead to an increase of items being wasted due to cross-contamination among items that are closely packed together.

Finally, in this paper we have used the CLAs and the cross-contamination example to demonstrate one possible application of our proximity-based ABS modelling approach. This approach can however be used to model underlying systems and environments (both in healthcare and in other domains) where entities need to be physically close to one another for interactions to take place. One example can be the propagation of forest fire; fire can spread from one tree to the next when, for example, there is physical proximity between the trees. Yet another example is the change in the spatial arrangement of items that are kept in physical proximity; this may be brought about when the items are being transported. Our future work involves extending the scenario presented and including such motion dynamics in order to perform rigid body ABS of items of cargo through time. More specifically, in order to enable this simulation, the agents will not only require the existing attributes pertaining to length, breadth, height, xPos, yPos and zPos, but they will also have to be bestowed with logic that enables Newtonian motion. In such a model the Newtonian motion that will be exhibited by the individual items will depend not only on the aforementioned properties, but also on new attributes like mass/weight, contact fiction, etc. The Newtonian motion can be modelled through use of a physics engine. Yet another requirement for modelling motion behaviour in items kept in close proximity is the detection of collision between objects. We have successfully experimented with Jinngine (SilcowitzHansen, 2010); however the integration of physics engine with our modelling environment remains a challenging future work!

\section{References}

Acharyulu, G. V. R. K. and Madhavedi, S. (2011). "Factors Contributing to Perishability in Traditional Fresh Produce Distribution System: A Study on Tomato and Banana Chains in Andhra Pradesh, India", International Journal of Advanced Economics and Business Management, 1(1):1-5.

Ackerley, N., Sertkaya, A. and Lange, R. (2010). "Food Transportation Safety: Characterizing Risks and Controls by Use of Expert Opinion", Food Protection Trends, 30(4): 212-222.

Ashby, B. H. (1995). "Protecting perishable foods during transport by truck", Handbook no. 669, US Department of Agriculture, Argicultural Marketing Service. Available online http://www.ams.usda.gov/AMSv1.0/getfile?dDocName=STELDEV3021003 [last accessed 29 June 2012].

Bagni, R., Berchi, R. and Cariello, P. (2002). "A comparison of simulation models applied to epidemics", Journal of Artificial Societies and Social Simulation, 5(3).

Bischoff, E.E. (2006). "3-D Packing of Items with Limited Load Bearing Strength", European Journal of Operational Research, 168(3): 952-966.

Bhat, R. V. (1988). "Mould deterioration of agricultural commodities during transit: problems faced by developing countries", International Journal of Food Microbiology, 7(3): 219-225.

Bunn, D.W., and Oliveira, F.S. (2001). "Agent-based simulation-an application to the new electricity trading", IEEE Transactions on Evolutionary Computation, 5(5): 493-503.

Carley, K.M., Fridsma, D.B., Casman, E., Yahja, A., Altman, N., Chen L-C., Kaminsky, B. and Nave D. (2006). "BioWar: Scalable Agent-Based Model of Bioattacks", IEEE Transactions on Systems, Man, and Cybernetics, Part A: Systems and Humans, 36(2): 252-265.

CASOS. (2012). Center for Computational Analysis of Social and Organizational Systems. School of Computer Science, Carnegie Mellon. Available online http://www.casos.cs.cmu.edu/ [Last accessed 30 June 2012].

Chen, H-K., Hsueh, C-F, and Chang, M-S. (2009). "Production scheduling and vehicle routing with time windows for perishable food products", Computers \& Operations Research, 36(7): 2311-2319.

Coburn, B.J., Wagner, B.G. and Blower, S. (2009). "Modelling influenza epidemics and pandemics: insights into the future of swine flu (H1N1)", BMC Medicine, 7: 30. 
Downing, T.E., Moss, S. and Pahl-Wostl, C. (2001), "Understanding Climate Policy Using Participatory Agent-Based Social Simulation", Lecture Notes in Computer Science, Volume 1979/2001, 127-140.

Dunham, J.B. (2005). "An Agent-Based Spatially Explicit Epidemiological Model in MASON”, Journal of Artificial Societies and Social Simulation. 9, 1.

Eidelson, B.M. and Lustick, I. (2004). "VIR-POX: An Agent-Based Analysis of Smallpox Preparedness and Response Policy", Journal of Artificial Societies and Social Simulation, 7, 3.

Emrich, S., Suslov, S. and Judex, F. (2007). "Fully Agent Based. Modellings of Epidemic Spread Using Anylogic". Proc. EUROSIM, 9-13 Sept. 2007, Ljubljana, Slovenia.

Eubank, S., Guclu, H., Kumar, A.V.S, Marathe, M., Srinivasan, A., Totoczkai, Z. and Wang, N. (2004). "Modelling disease outbreaks in realistic urban social networks", Nature, 429: 180-184.

Ferguson, N.M, Cummings, D.A.T, Fraser, C., Cajka, J.C., Cooley, P.C. and Burke, D.S. (2006). "Strategies for mitigating an influenza pandemic", Nature, 442: 448-52.

Gordon, T.J (2003). "A simple agent model of an epidemic", Technological Forecasting and Social Change, 70(5): 397-417.

Gotts, N.M., Polhill, J.G. and Law, A.N.R. (2003). "Agent-Based Simulation in the Study of Social Dilemmas", Artificial Intelligence Review, 19(1): 3-92.

Halloran, M.E., Longini, I.M. Jr., Nizam, A. and Yang, Y. (2002) "Containing bioterrorist smallpox", Science, 298, 5597: 1428-1432.

Halloran, M.E., Ferguson, N.M., Eubank, S., Longini, I.M. Jr., Cummings, D.A., et al. (2008). "Modelling targeted layered containment of an influenza pandemic in the United States", Proceedings of the National Academy of Sciences of the United States of America,105, 12: 4639-4644.

Harding, P. L., and Dyer (jr.), R. M. M. (1942). "Effect of thinning on some of the phyical and chemical characters of valencia oranges". In Proceeding of the Florida State Hortricultural Society, vol 55: 34-38. Available online http://www.fshs.org [last accessed 29 June 2012].

Hotchkiss, J.R., Strike, D.G., Simonson, D.A., Broccard, A.F. and Crooke, P.S. (2005). "An agent-based and spatially explicit model of pathogen dissemination in the intensive care unit", Critical Care Medicine, 33(1): 168-176.

Katsaliaki, K. and Mustafee, N. (2011). "Applications of Simulation Research within the Healthcare Context", Journal of the Operation Research Society, 62(8): 1431-1451.

Macal, C.M. and North, M.J. (2006), "Tutorial on Agent-Based Modelling and Simulation Part 2: How to Model with Agents", In Proceedings of the 2006 Winter Simulation Conference, pp. 73-83.

Macal, C. M. and North, M.J. (2010). "Tutorial on agent-based modelling and simulation”, Journal of Simulation, 4(3): 151-162.

Mustafee, N., Katsaliaki, K. and Taylor, S.J.E. (2010). "Profiling Literature in Healthcare Simulation", SIMULATION: Transactions of the Society of Modelling and Simulation International, 86(8-9): 543-558.

Patlolla, P., Gunupudi, V., Mikler, A.R. and Jacob, R.T. (2004). "Agent-Based Simulation Tools in Computational Epidemiology", In Proceedings of the International Conference on Innovative Internet Community Systems (I2CS '04), pp. 212-223.

Pisinger, D. (2002). "Heuristics for the container loading problem", European Journal of Operational Research, 141(2): 292-382.

Raberto, M., Cincottia, S., Focardib, S.M. and Marchesic, M. (2001). "Agent-based simulation of a financial market", Physica A: Statistical Mechanics and its Applications, 299(1-2): 319-327.

Robinson, E. A. and McLeod, J. (1989). "Packaging device and method", United States Patent, Patent Number: 4807424, Date of Patent: Feb 28, 1989. Available online http://www.google.com/patents/US4807424 [last accessed 29 June 2012].

Shaliz, C. R. 2006. "Methods and techniques of complex systems science: An overview." In Complex Systems Science in Biomedicine, Edited by T. S. Deisboeck and J. Y. Kresh, 33-114. New York: Springer.

Sibbel, R. and Urban, C. (2001). "Agent-based modelling and simulation for hospital management." Cooperative Agent: Applications in the Social Sciences, Edited by N. J. Saam and B. Schmidt, 183-202. The Netherlands: Kluwer Academic Publishers., 
Song, J., Haasb, C. T. and Caldas, C. H. (2007). "A proximity-based method for locating RFID tagged objects", Advanced Engineering Informatics, 21(4):367-376.

Stainsby, H., Taboada, M. and Luque, E. (2009). "Towards an Agent-Based Simulation of Hospital Emergency Departments". In Proceedings of the 2009 IEEE International Conference on Services Computing, pp. 536 - 539.

Silcowitz-Hansen, M. (2010). "Jinngine - a Physics Engine Written In Java". Available online http://code.google.com/p/jinngine/ [last accessed 29 June 2012].

Tournas, V. H. and Katsoudas, E. (2005). "Mould and yeast flora in fresh berries, grapes and citrus fruits", International Journal of Food Microbiology, 105(1):11-17.

Vaught, J.B. (2006). "Blood collection, shipment, processing, and storage", Cancer Epidemiol Biomarkers Prev, 15(9): 1582-4.

Wascher, G., Haubner, H. and Schumann, H. (2007). "An improved typology of cutting and packing problems", European Journal of Operational Research, 183(3): 1109-1130.

World Health Organisation. (2002). "Guidelines on Prevention and Control of Hospital Associated Infections", Regional Office for South-East Asia, New Delhi: WHO.

World Health Organisation. (2003). "Guide to good storage practices for pharmaceuticals", WHO Technical Report Series, No. 908, Geneva, Switzerland; WHO.

World Health Organisation. (2009). “WHO Guiding Principles on Human Organ Transplantation”, Report of the Regional Meeting, Malaysia: WHO Western Pacific Region.

XJ Technologies. (2011). "AnyLogic Agent Based Modelling - Help Files. Available online http://www.xjtek.com/anylogic/help (last accessed 3rd November 2011). 


\section{Greyscale Figures}

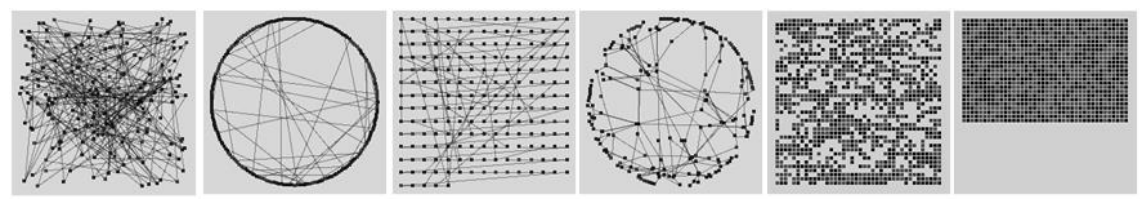

Figure 4: AnyLogic-defined arrangement of agents (from left to right): Random layout, Ring layout, Arranged layout, Spring Mass layout, Random layout (discrete space), Arranged layout (discrete space) (XJ Technologies, 2011). 


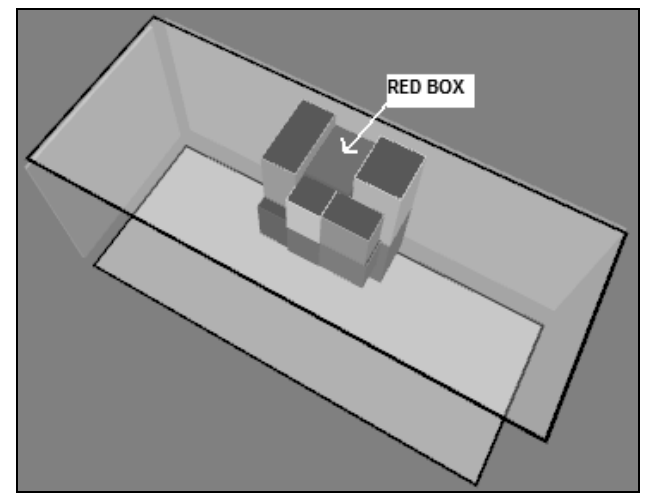

Figure 5: Establishing inter-agent relationship based on physical proximity between agents 


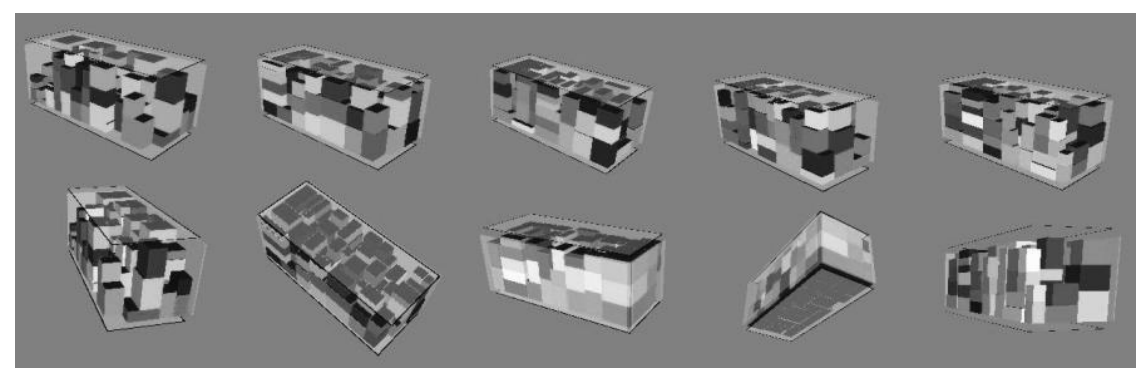

Figure 6: $\mathrm{CPO}$ algorithm being used to generate various layouts for the location-aware agents and to create inter-agent relationships between them. The agents are represented by the individual boxes in the confined space. The data is visualised using AnyLogic. 


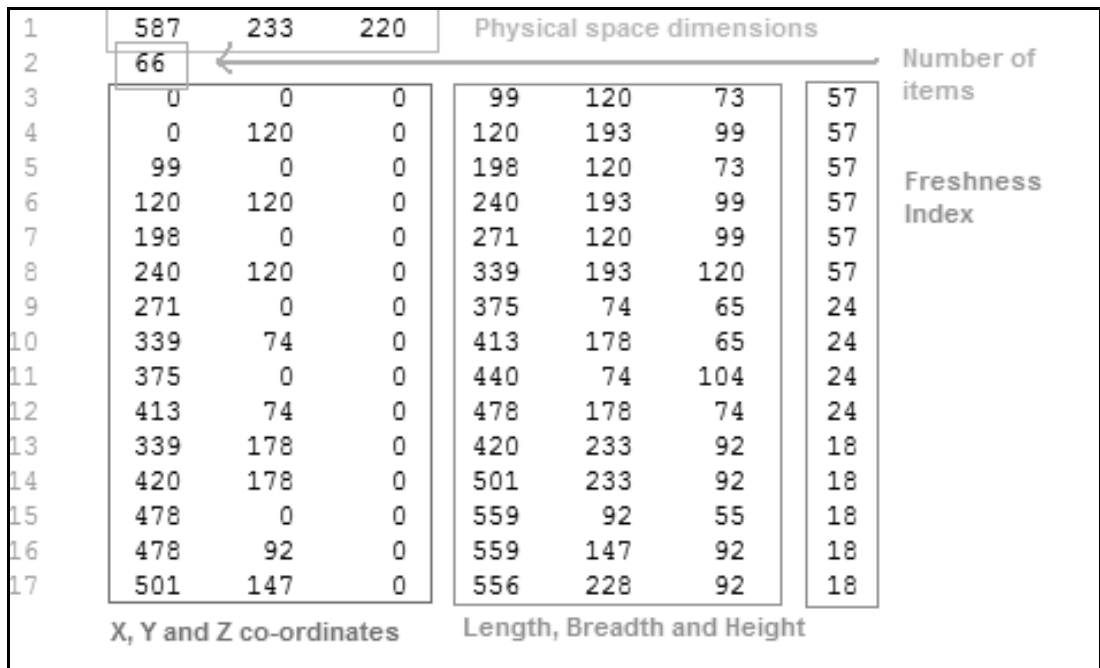

Figure 4: Output of the Container Loading Algorithm (Bischoff, 2006); used as input for the creation of inter-agent relationship in our ABS model 


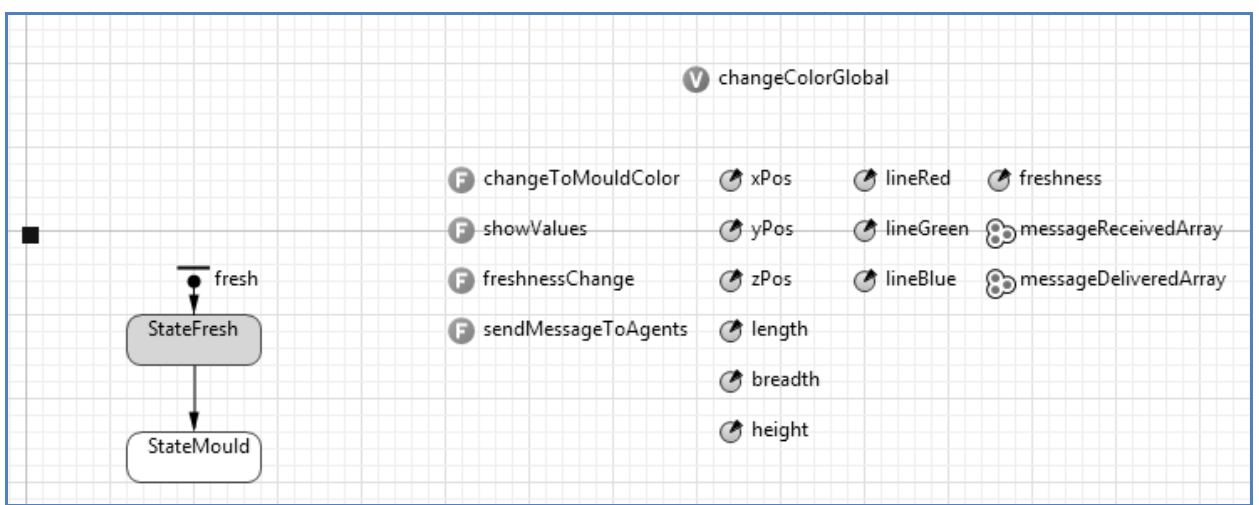

Figure 5: Agent state chart (left) and the variables and functions used to realise the model 


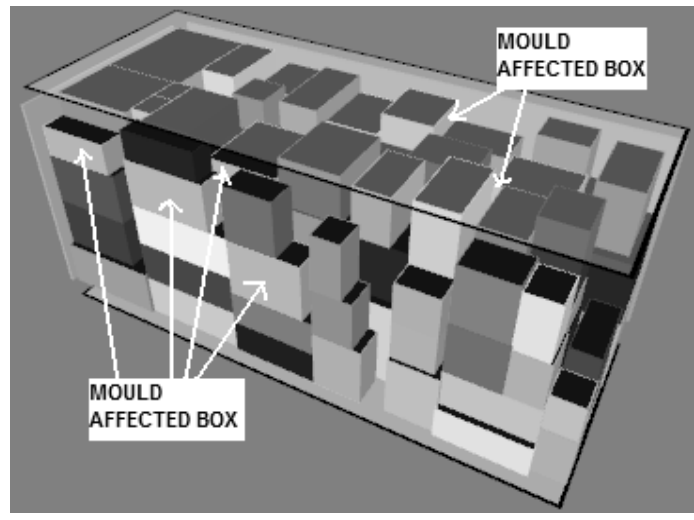

Figure 6: Boxes get cross-contaminated through the propagation of mould as the simulation advances through time 


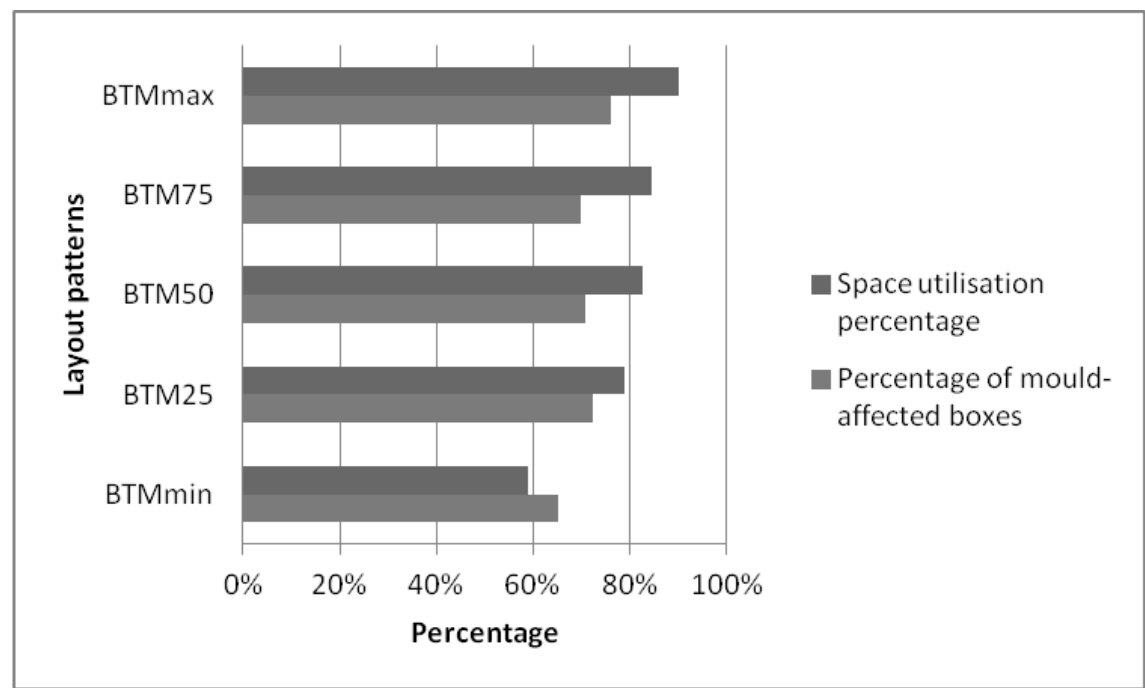

Figure 7: Percentage of mould-affected boxes for different space utilisation percentages 